\title{
Correction: Comparison of the handheld RETeval ERG system with a routine ERG system in healthy adults and in paediatric patients
}

\author{
Perry Carter (D) Arabella Gordon-Reid • Fatima Shawkat • Jay E. Self $(D$
}

Published online: 30 October 2020

(C) The Author(s), under exclusive licence to The Royal College of Ophthalmologists 2020

Correction to: Eye

https://doi.org/10.1038/s41433-020-01221-2

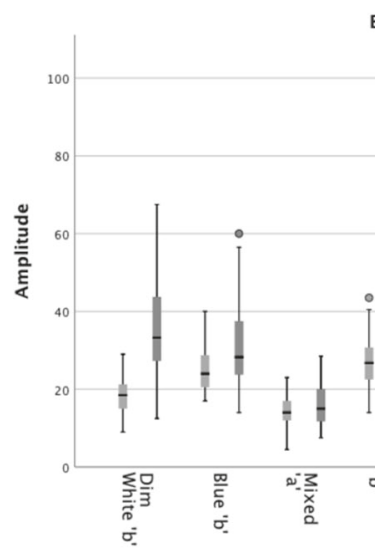

Boxplot of amplitude

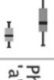

$\frac{1}{1} \mathrm{I}$


The original Article was corrected shortly after publication because Fig. 1 previously had typographical errors. Please find the correct version below and in the original Article.

\begin{tabular}{|c|c|c|c|c|c|c|c|c|}
\hline \multicolumn{2}{|c|}{$\begin{array}{l}\text { Reference range } \\
\text { Amplitude ( } \mu \mathrm{V})\end{array}$} & $\begin{array}{l}\text { Mixed 'a' } \\
\text { wave }\end{array}$ & $\begin{array}{l}\text { Mixed 'b' } \\
\text { wave }\end{array}$ & $\begin{array}{l}\text { Dim White } \\
\text { 'b' wave }\end{array}$ & $\begin{array}{l}\text { Dim Blue 'b' } \\
\text { wave }\end{array}$ & $\begin{array}{l}\text { Photopic 'a' } \\
\text { wave }\end{array}$ & $\begin{array}{l}\text { Photopic 'b' } \\
\text { wave }\end{array}$ & Flicker \\
\hline \multirow[t]{2}{*}{ Standard } & Mean & 16.3 & 46.7 & 35.8 & 31.7 & 10.6 & 24.9 & 15.0 \\
\hline & $2.5^{\text {th }}-97.5^{\text {th }}$ & $8.1-29.0$ & $24.0-78.0$ & $19.1-61.0$ & $18.0-56.0$ & $5.0-22.0$ & $11.0-43.9$ & $6.0-29.9$ \\
\hline \multirow{2}{*}{$\begin{array}{c}\text { RETeval } \\
\text { (trolands) }\end{array}$} & Mean & 14.2 & 27.1 & 18.1 & 25.4 & 6.2 & 20.8 & 23.7 \\
\hline & $2.5^{\text {th }}-97.5^{\text {th }}$ & $5.1-23.0$ & $16.0-41.0$ & $10.0-29.0$ & $17.0-39.0$ & $3.0-10.0$ & $11.0-30.0$ & $11.0-36.1$ \\
\hline \multicolumn{2}{|c|}{$\begin{array}{l}\text { Reference range } \\
\text { Peak time (ms) }\end{array}$} & $\begin{array}{l}\text { Mixed 'a' } \\
\text { wave }\end{array}$ & $\begin{array}{l}\text { Mixed 'b' } \\
\text { wave }\end{array}$ & $\begin{array}{l}\text { Dim White } \\
\text { 'b' wave }\end{array}$ & $\begin{array}{l}\text { Blue 'b' } \\
\text { wave }\end{array}$ & $\begin{array}{l}\text { Photopic 'a' } \\
\text { wave }\end{array}$ & $\begin{array}{l}\text { Photopic 'b' } \\
\text { wave }\end{array}$ & Flicker \\
\hline \multirow[t]{2}{*}{ Standard } & Mean & 16.5 & 40.1 & 50.0 & 52.8 & 15.1 & 34.2 & 32.1 \\
\hline & $2.5^{\text {th }}-97.5^{\text {th }}$ & $12.0-21.0$ & $36.0-46.0$ & $39.1-60.0$ & $44.1-61.0$ & $11.0-21.0$ & $29.1-41.9$ & $27.1-37.0$ \\
\hline \multirow{2}{*}{$\begin{array}{c}\text { RETeval } \\
\text { (trolands) }\end{array}$} & Mean & 13.1 & 38.4 & 63.7 & 57.4 & 10.5 & 29.2 & 25.5 \\
\hline & $2.5^{\text {th }}-97.5^{\text {th }}$ & $10.0-25.0$ & $32.0-49.0$ & $50.0-86.0$ & $48.0-71.0$ & $6.0-14.0$ & $25.0-33.0$ & $24.0-32.0$ \\
\hline
\end{tabular}

Fig. 1 